\title{
Database Constraints Applied to Metabolic Pathway Reconstruction Tools
}

\author{
Jordi Vilaplana, ${ }^{1}$ Francesc Solsona, ${ }^{1}$ Ivan Teixido, ${ }^{1}$ Anabel Usié, ${ }^{2}$ \\ Hiren Karathia, ${ }^{3}$ Rui Alves, ${ }^{3}$ and Jordi Mateo ${ }^{1}$ \\ ${ }^{1}$ Computer Science Department \& INSPIRES, University of Lleida, Jaume II 69, 25001 Lleida, Spain \\ ${ }^{2}$ CEBAL, IPBeja Campus, 7800 Beja, Portugal \\ ${ }^{3}$ Department of Basic Medical Sciences \& IRBLleida, Edifici Recerca Biomedica I, Universitat de Lleida, \\ Avenida Rovira Roure 80, 25198 Lleida, Spain \\ Correspondence should be addressed to Francesc Solsona; francesc@diei.udl.cat
}

Received 8 February 2014; Revised 7 July 2014; Accepted 8 July 2014; Published 17 August 2014

Academic Editor: Antonio Puliafito

Copyright (c) 2014 Jordi Vilaplana et al. This is an open access article distributed under the Creative Commons Attribution License, which permits unrestricted use, distribution, and reproduction in any medium, provided the original work is properly cited.

\begin{abstract}
Our group developed two biological applications, Biblio-MetReS and Homol-MetReS, accessing the same database of organisms with annotated genes. Biblio-MetReS is a data-mining application that facilitates the reconstruction of molecular networks based on automated text-mining analysis of published scientific literature. Homol-MetReS allows functional (re)annotation of proteomes, to properly identify both the individual proteins involved in the process(es) of interest and their function. It also enables the sets of proteins involved in the process(es) in different organisms to be compared directly. The efficiency of these biological applications is directly related to the design of the shared database. We classified and analyzed the different kinds of access to the database. Based on this study, we tried to adjust and tune the configurable parameters of the database server to reach the best performance of the communication data link to/from the database system. Different database technologies were analyzed. We started the study with a public relational SQL database, MySQL. Then, the same database was implemented by a MapReduce-based database named HBase. The results indicated that the standard configuration of $M y S Q L$ gives an acceptable performance for low or medium size databases. Nevertheless, tuning database parameters can greatly improve the performance and lead to very competitive runtimes.
\end{abstract}

\section{Introduction}

Our group developed two biological applications, BiblioMetReS (http://metres.udl.cat/index.php/2-uncategorised/ 13-biblio-metres-paper-published) [1] and Homol-MetReS (http://homolmetres.udl.cat/prod/) [2], respectively, acronyms of Bibliomet- and Homolog-based metabolic network reconstruction server. These applications rely on an inhouse relational database [2]. The database was built by matching the KEGG (http://www.genome.jp/kegg/) gene names to their NCBI (http://www.ncbi.nlm.nih.gov) names and synonyms. The database includes full gene names and synonyms tables for approximately 1,500 organisms with fully sequenced genomes. The database was implemented using MySQL (http://www.mysql.com/), one of the most popular open-source databases, mainly managed with $S Q L$ (structured query language), a standard programming language designed for managing data held in a relational database.

Biblio-MetReS is a user-friendly tool implemented in Java, which does on-the-fly analyses of the full text of scientific documents that are freely available on the Internet, and uses that analysis for the automated reconstruction of gene/protein networks. Such an on-the-fly approach leads to high runtimes, because the analysis of scientific documents is very resource consuming. To circumvent this high runtime, preprocessing of the documents [3-5] is typically used. Preprocessing of texts/documents consists of analyzing the documents offline, extracting the relevant information, and creating an adequate table to contain that information inside the database.

Homol-MetReS is a web application that permits simultaneous large-scale reannotation, functional integration, and automatic comparisons of metabolic networks on a multiple 
full genome scale. It automates comparisons that would otherwise be done almost manually, for example, using PathBlast [6], KEGG [7], or MetaCYC [8]. The functionalities in Homol-MetReS that have the highest needs for processing power and database access optimization are the comparison between fully sequenced genomes and the classification of large numbers of homologous proteins according to specific biological process categories. As in the case of Biblio-MetReS, database access is a bottleneck in the runtime of the server.

The system architecture (a data-center) hosting both applications presents a single access point for the computing needs of the users being served, like the ones described in [ 9 , 10]. As stated in [11], most current data-center infrastructures consist of services that are offered by a web browser anywhere in the world. A service request sent by a user is transmitted to a server running a web service, which is associated with an SLA (service-level agreement). An SLA is a contract that a customer and a service provider have negotiated and agreed to. In such a contract, the customer only pays for the resources and services used, according to negotiated QoS (quality of service) requirements at a given price. Job runtime is perhaps the most important performance index in a cloud computing context [12], and it largely depends on the database system.

The requirement to perform compute-intensive analytics on (semi)structured bulk datasets has pushed SQL-like centralized databases to their limits [13]. This fact, along with the highly parallel nature of these tasks, has led to the development of horizontal scalable, distributed nonrelational data stores, called NoSQL (http://nosql-databases.org/) databases. Google's Bigtable [14], Amazon's Dynamo [15], Facebook's Cassandra [16], LinkedIn's Voldemort [17], and HBase are a representative sample of such systems.

In favor of scalability and high availability, NoSQL systems serve a dual purpose: they can efficiently store and index NoSQL sets arbitrarily while enabling a large number of concurrent user requests. Among other reasons, NoSQL systems may not be the optimal solution when data-centers have no need to deal with large datasets. However, in order to provide the overall system with a negotiated SLA, optimizing the current SQL database system may be a challenge. In [18], the authors dealt with the challenge of optimizing a $M y S Q L$ database for novelty detection, which is the process of singling out novel information from a given set of text documents, in order to optimize the database tables for up to 10 million records. In [19], the authors also used database optimization and SQL tuning in order to achieve better performance levels for health monitoring systems. In this paper, in addition to these proposals we propose the application of preprocessing in order to guarantee a negotiated SLA in web-based systems with a MySQL biological database. Preprocessing of texts/documents consists of analyzing the documents offline, creating a database where the interaction information for each document is stored and can be quickly accessed [3-5]. Response time to client requests is shorter because it just depends on the elapsed time in accessing the database.

In the Results section of the present work, comparisons between optimized and nonoptimized $M y S Q L$ databases were performed. We found that performing specific
TABle 1: Main differences between Biblio-MetReS (B), iHOP (i), String (S), and Laitor (L).

\begin{tabular}{lllll}
\hline & B & i & S & L \\
\hline Multiplatform & X & X & X & \\
Gene correlation in sentences & X & X & & X \\
Gene correlation in paragraphs & X & & & \\
Preprocessing & X & X & X & \\
Statistical method: $P$ value & X & & & \\
Statistical method: cooccurrence & X & X & X & X \\
List of synonyms & X & X & X & X \\
Interaction extraction between genes & X & X & & X \\
Semantic interaction understanding & & X & & X \\
Graphical representation & X & X & X & \\
\hline
\end{tabular}

optimization of some database server parameters led to a significant performance increase without having to migrate the entire system to a different approach, like a NoSQL system. We show this by comparing the MySQL database with a NoSQL one, implemented with MapReduce (http://research.google.com/archive/mapreduce.html) and HBase.

\section{Related Work and Motivation}

There are important tools in the literature for the automatic identification of cooccurrence of genes/proteins from Internet to assist in the reconstruction of molecular circuits by inferring the gene/protein entities and their cooccurrence, such as Laitor [3], iHOP [4], String [5], and Biblio-MetReS, released in March 2011 [1] by our group.

Table 1 summarizes the main differences between these tools. Biblio-MetReS is the only tool able to search for gene cooccurrences in sentences, paragraphs, and the overall document. This allows Biblio-MetReS to better assess the interactions between a pair of genes. In addition to the cooccurrence statistic methods, Biblio-MetReS also provides the $P$ value associated with cooccurrence, reducing the likelihood of false negatives or false positives when looking for genes/proteins in the texts/documents.

The main drawback of Biblio-MetReS is the elevated elapsed time processing the documents downloaded from Internet to extract gene/protein interactions. iHOP, String, and others (see Table 1 from [1] and references therein) are quicker in responding to client requests because they implement the preprocessing of information (documents) technique offline. Bilblio-MetReS also incorporates that, but all work is performed online. In this paper we deal with the database efficiency by tuning the MySQL parameters and incorporating the preprocessing.

Most current data-centers architecture consists of services that are offered and delivered through a service center that can be accessed from a web browser anywhere in the world (Figure 1).

In the Biblio-MetReS application, a desktop application acts as a client of the web service. In the case of HomolMetReS, the client is the web browser itself. Requests delivered to the data-center are associated with an SLA. Our work 


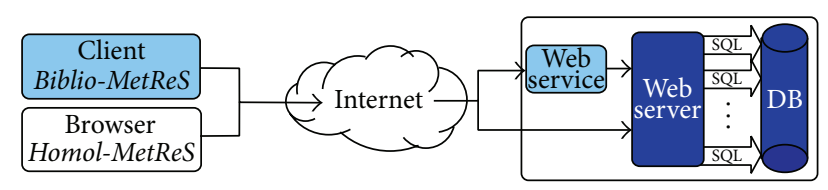

FIGURE 1: Cloud computing architecture.

is focused on guaranteeing the SLA (service-level agreement). On this occasion, the database performance was the main focus of our research. In doing so, we propose some solutions to provide a high level of QoS, thus determining a means to fix the SLA in accessing the database for the particular case of our subject applications.

The use of model organisms for research is a hallmark of scientific endeavour (e.g., [20]). The accumulation of fully sequenced genomes [21] and the advances in comparative genomics [22, 23] and computational systems biology [24] allow us to develop strategies that compare the protein or gene networks involved in the process of interest in order to establish similarities. These similarities can be used to predict, to a first approximation, the accuracy of extrapolating the behavior of specific processes between organisms. Testing this idea requires a thorough analysis of the molecular circuits in a well-known model organism and a comparison of these circuits to those in other living beings. In fact, a gap exists in systematically establishing how close different organisms are with respect to a given process, before choosing one of them as a model for studying that process [2]. Provided the Biblio-MetReS database of fully sequenced genomes, HomolMetReS implements innovative functionality in such research field. Thus, as in the case of Biblio-MetReS, we are interested in providing Homol-MetReS with SLA guarantees.

\section{Biblio-MetReS}

Biblio-MetReS is a Java client-server application which needs a database in order to manage biological data efficiently, and more specifically information about organisms, genes/proteins, the processes in which the genes are involved, and the relationships between all these entities. Biblio-MetReS searches Internet for documents with datamining techniques and operates as follows. Users must register to login in to Biblio-MetReS. After login, users must choose an organism to work with. The application loads all the genes in the database for the selected organism. Once this is loaded, the user is presented with the main window (Figure 2), where s/he can select data sources as well as genes to search for in those data sources across Internet. The data sources are as follows: General Search Engines (Yahoo (http://yahoo.com/), Live Search (http://www.bing.com/), Ask (http://www.ask.com/), Answers (http://www.answers .com/topic/statistics), Altheweb (http://altheweb.com/), and Lycos (http://www.lycos.com/)), literature databases (Medline (http://www.nlm.nih.gov), PubMed (http://www.ncbi .nlm.nih.gov/pubmed), Biomed Central (http://www.biomedcentral.com/), PLoS (http://www.plos.org/), Bentham (http://www.benthampapers.ucl.ac.uk/), Highwire (http:// highwire.stanford.edu/), SCOPUS (http://www.scopus.com/), and Elsevier (http://www.elsevier.com/)), and journals ( $\mathrm{Na}$ ture (http://www.nature.com/), Science (http://www.sciencemag.org/), Cell (http://www.cell.com/), PNAS (http://www .pnas.org/), and BMC Systems Biology (http://www.biomedcentral.com/bmcsystbiol)).

Once the choices are made and the search has started, the tool identifies and downloads the documents from the selected data sources that contain the gene names used in the search query. Documents may be in HTML, PDF, or ASCII formats. Each document is then parsed to identify all other genes and gene synonyms (that were retrieved from the database) from the organism of interest that are also mentioned in the documents. A similar workflow is used to analyze biological processes in the documents. Entity cooccurrence patterns are analyzed and represented in several ways. For example, cooccurrence patterns can be represented using a $2 \mathrm{D}$ graph of the gene cooccurrence. The whole analysis process is done using a mixed strategy that combines newly found documents on the fly with preprocessed information retrieved from the database if a document has been found in previous searches. Basically, the performance of the tool depends on its effectiveness in processing documents and the online access to the database. Reference [1] provides the details on how cooccurrence is analyzed in documents.

\section{Homol-MetReS}

Proper functional identification of genes on a full genome scale for all organisms with fully sequenced genomes is only possible by transferring the functional information that is available for proteins from other organisms with fully sequenced genomes. Homol-MetReS is a web application that permits the functional information of a model organism to be extrapolated to other organisms where that process or circuit is hard to study or there is insufficient information.

Homol-MetReS provides three main functionalities. First, users can (re)annotate the function of each of the proteins in the proteome of an organism of interest with respect to many different classifications of biological functions. Although interesting, this functionality is independent of database performance, as the process is done via the analysis of preexisting text files and the creation of new ones to store transferred information.

Second, users can automatically compare the sequences of the individual proteins from their proteome of interest to those of the full proteome from more than 1,200 other organisms that have fully sequenced and annotated genomes. Functional information from one organism can be transferred to another by the user, based on sequence homology. The process for doing so is illustrated in Figure 3. One of the bottlenecks in the computational process underlying this functionality is access to the database. This is so because many requests must be sent (in parallel when possible) to the database in order to compare the full proteome of an arbitrary number of organisms. These comparisons between organisms must be done within a reasonable time. We deal with the operations related to this kind of comparisons in the Results section. The database operations used in this functionality 


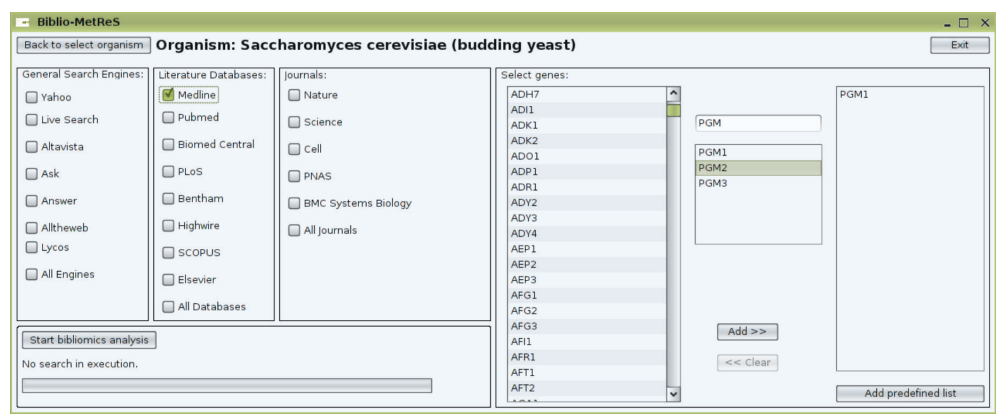

Figure 2: Bilio-MetReS. Main window.

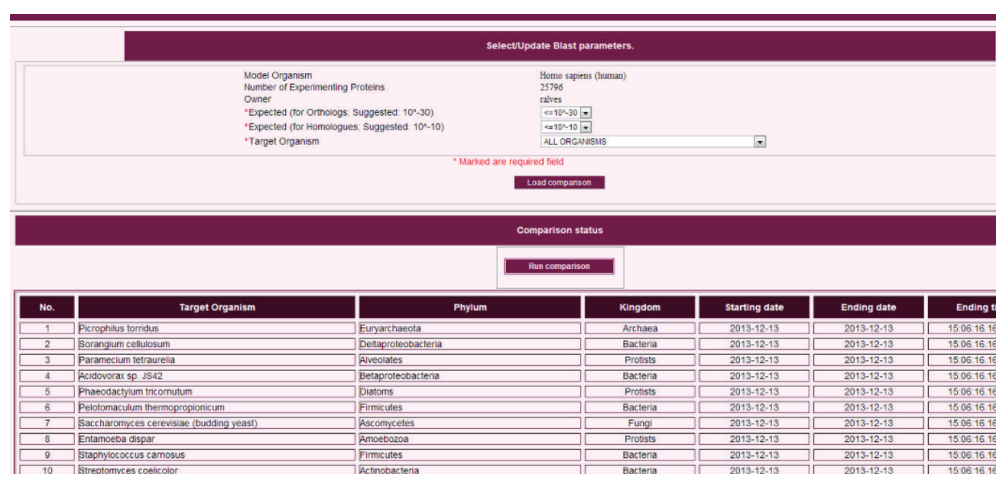

FIGURE 3: Homol-MetReS.

can mainly be classified as follows: (a) operations retrieving sequence information for the complete proteomes of any set of organisms contained in the database, (b) operations comparing information data of multiple organisms, and (c) operations to create temporary tables that contain information about the comparative analysis of the proteins between the organisms being analyzed. Third, the tables created in (c) are then used to represent heat maps that allow users to visually compare the similarity between the sets of proteins involved in specific biological processes in all the organisms being analyzed. Neither database access nor I/O is limiting for this functionality. Thus, its performance analysis was discarded in the Results section (Section 6).

\section{Database Optimization}

In order to optimize the interaction between Biblio- and Homol-MetReS and the database server, we designed a cloud architecture made up of 3 virtual machines. The first two contain the Biblio- and Homol-MetReS servers, respectively. The third virtual machine contains the database server. These three virtual machines were implemented using the OpenStack (http://www.openstack.org/software) framework. OpenStack is able to create as many virtual machines as needed in a dynamic manner. However, the main reason for choosing OpenStack was its freeware availability.

We now present the shared database used by BiblioMetReS and Homol-MetReS, highlighting the most important tables. Then, an analysis of the improvements and optimization done on the database parameters is performed.

Figure 4 shows a basic schema of the current relational database, implemented by means of MySQL.

According to the relational database definition, our database consists of a collection of tables organized according to the relational model with defined relationships with each other. There are the tables corresponding to organisms, genes, processes, and documents. These tables contain the information most frequently accessed by the applications and, therefore, the ones we focus on. Note that, in Figure 4, the tables corresponding to each of the different organisms present in the database are not explicitly listed. This is because there is one table for each defined organism and there are currently more than 1,200 different organisms in the database. In addition, although genes are organism-specific, biological processes are general and apply to all organisms. The relationship between genes and biological processes is also stored in organism-specific tables.

Also, there are other secondary tables in Figure 4 that are defined in order to support the applications. These tables include, for example, such user information as the country and login name. These tables are not considered, as they lack interest for our current purposes.

Table 2 shows an example of one of the tables that contains information about the proteins coded for in the genome of a fully sequenced organism contained in the central $M y S Q L$ database. All such tables follow the same field structure, as shown in Table 2, where XXX_ORG_ID corresponds to the prefix of the organism. 


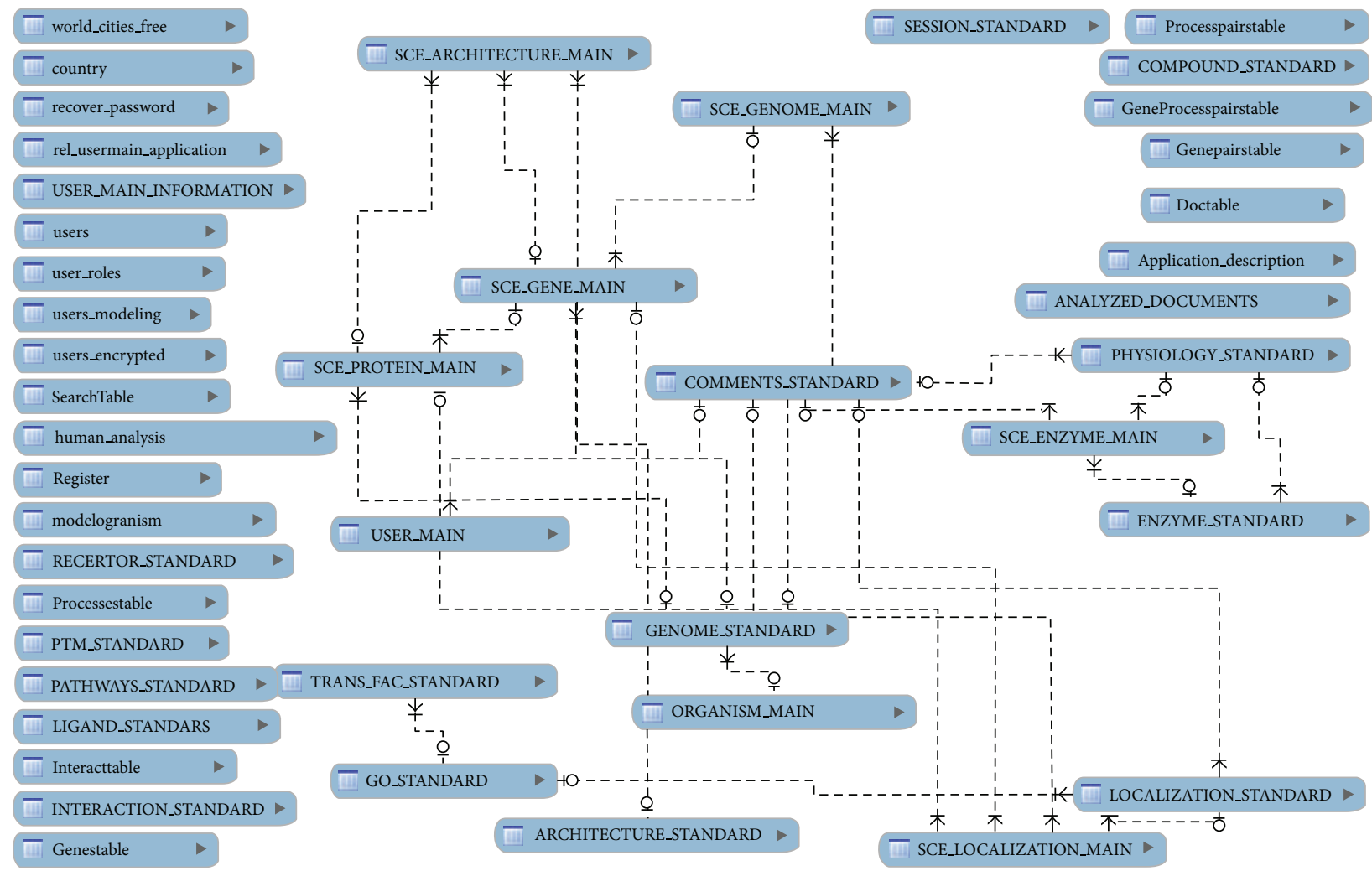

Figure 4: Simplified database schema.

TABLE 2: Generic organism table description.

\begin{tabular}{|c|c|c|c|c|}
\hline Field & Type & Null & Key & Default \\
\hline XXX_isoform_id & char(20) & $\mathrm{NO}$ & PRI & \\
\hline XXX_protein_id & $\operatorname{char}(20)$ & YES & & NULL \\
\hline XXX_gene_id & $\operatorname{char}(20)$ & YES & & NULL \\
\hline XXX_org_id & $\operatorname{char}(20)$ & YES & & NULL \\
\hline XXX_kegg_id & $\operatorname{char}(30)$ & YES & & NULL \\
\hline isoform_no & $\operatorname{int}(5)$ & YES & MUL & NULL \\
\hline XXX_gene_symbol & $\operatorname{varchar}(50)$ & YES & & NULL \\
\hline XXX_prot_name & $\operatorname{varchar}(500)$ & YES & & NULL \\
\hline XXX_prot_synonym & $\operatorname{varchar}(500)$ & YES & & NULL \\
\hline ncbi_ref_id & $\operatorname{char}(25)$ & YES & & NULL \\
\hline gi_number & $\operatorname{char}(25)$ & YES & & NULL \\
\hline orf_start & $\operatorname{int}(5)$ & YES & & NULL \\
\hline orf_end & $\operatorname{int}(5)$ & YES & & NULL \\
\hline isoform_sequence & blob & YES & & NULL \\
\hline isoform_length & $\operatorname{int}(5)$ & YES & & NULL \\
\hline isoform_mol_wt & $\operatorname{int}(8)$ & YES & & NULL \\
\hline comments & $\operatorname{char}(200)$ & YES & & NULL \\
\hline status & $\operatorname{char}(50)$ & YES & & NULL \\
\hline
\end{tabular}

Table 3 shows the description of the process table (Processestable) identified by Biblio-MetReS during the analysis of scientific documents. This table is general and stores information about biological processes associated with genes
TABLE 3: Generic process.

\begin{tabular}{lcllc}
\hline Field & Type & Null & Key & Default \\
\hline Processes & varchar(100) & NO & PRI & NULL \\
Dockey & $\operatorname{int}(11)$ & NO & PRI & 0 \\
organism & $\operatorname{varchar(100)}$ & NO & PRI & NULL \\
individualOC & $\operatorname{int}(10)$ & NO & & NULL \\
typeProcess & $\operatorname{int}(10)$ & NO & PRI & NULL \\
\hline
\end{tabular}

from one of the organisms with fully sequenced genomes currently included in the central database (e.g., Saccharomyces cerevisiae, Homo sapiens, Escherichia coli K12 MG1655, or Drosophila melanogaster). The XXX_isoform_id column identifies which isoform of the gene is considered if the gene codes for more than one such isoform. The status column indicates whether the isoform has been experimentally confirmed or only predicted through bioinformatics analysis.

Table 4 summarizes the genes table (Genestable), which stores information regarding the genes that are found by Biblio-MetReS during the analysis of scientific documents. The processes, typeofprocesses, and organism columns connect the information found in the literature to the relevant organism and process contained in the database, while the other columns identify the document in which the process was identified.

Table 5 shows the description of the documents table (Doctable), which stores statistical information about the 
TABLE 4: Generic genes table description.

\begin{tabular}{lcllc}
\hline Field & Type & Null & Key & Default \\
\hline Genes & varchar(100) & NO & PRI & \\
Dockey & $\operatorname{int}(11)$ & NO & PRI & 0 \\
organism & $\operatorname{varchar(100)}$ & NO & PRI & \\
individualOC & $\operatorname{int}(10)$ & NO & & NULL \\
\hline
\end{tabular}

TABle 5: Document.

\begin{tabular}{lclll}
\hline Field & Type & Null & Key & Default \\
\hline Dockey & int(11) & NO & PRI & NULL \\
title & text & YES & & NULL \\
authors & text & NO & & NULL \\
url & text & YES & NULL \\
doi & $\operatorname{varchar}(80)$ & YES & NULL \\
pmcid & $\operatorname{varchar(80)}$ & YES & NULL \\
pmid & $\operatorname{varchar}(80)$ & YES & NULL \\
benthamid & $\operatorname{varchar}(80)$ & YES & NULL \\
cellid & $\operatorname{varchar}(80)$ & YES & NULL \\
bmcid & $\operatorname{varchar}(80)$ & YES & NULL \\
nSentence & int(10) & NO & NULL \\
nParagraph & int(10) & NO & NULL \\
\hline
\end{tabular}

documents found by Biblio-MetReS in Internet in the datamining search phase. The Dockey field is the unique ID of each document in the database. All other fields have information required to calculate mutual information for gene/protein cooccurrence in documents.

We now present the optimization we performed to the database. To facilitate understanding, the modifications related to Biblio- and Homol-MetReS are explained separately in the following sections.

5.1. Biblio-MetReS Optimization. Incoming documents found on the Internet are individually analyzed by BiblioMetReS. Once a document has been analyzed, the results are stored in the database using the tables described above, among others. Subsequent searches that identify the same document will retrieve the preprocessed statistics stored in the database, thus avoiding repeating the timeconsuming on-the-fly analysis. This process is known as preprocessing.

By implementing preprocessing we greatly decrease the time spent by the Biblio-MetReS application repeating the processing of documents. In contrast, much access to the database must be performed. On average, the database is accessed twice per new document to store statistical information for future use and 5 times per document to retrieve the statistical information from preprocessed documents when this information is required in subsequent searches.

Improvements in the database have little effect on the performance of Biblio-MetReS, as we will see in the Results section (Section 6). Because of this, we present and discuss the improvements made in database access in the following section, Homol-MetReS Optimization, where the gains are more significant.

5.2. Homol-MetReS Optimization. Several configuration parameters were adjusted to improve the database performance relating to the Homol-MetReS functionality. These parameters mainly affect the query caching done by the database server, hence improving query requests that will be executed more than once in a relatively short period of time.

These performance parameters were obtained using the MySQLTuner (https://github.com/major/MySQLTuner-perl) tool, which is a Perl (http://www.perl.org/) script that allows us to review a $M y S Q L$ database and perform adjustments in order to increase performance and stability.

The main running parameters related to optimizing database performance can be obtained by executing the MySQLTuner benchmark, and they are as follows: query_cache_size, table_open_cache, key_buffer_size, join_buffer_size, query_cache_limit, and innodb_buffer_pool_size. The query_cache_size parameter determines the amount of memory allocated to caching query results. If set to 0 , it disables the query cache. The optimal value depends on the system architecture. If set too high, it can provoke lock contention issues. The table_open_cache parameter determines the number of open tables for all threads. The number of file descriptors required by the MySQL server increases with table_open_cache. The key_buffer_size parameter determines the size of the buffer used for index blocks. The key buffer is also known as the key cache. The value of this variable indicates the amount of memory requested. Internally, the server allocates as much memory as possible up to this amount. It increases the value to obtain better index handling for all reads and multiple writes. The join_buffer_size parameter determines the size of each join buffer. The query_cache_limit parameter determines the maximum size of individual query results that can be cached.

\section{Results}

In this section, we present a set of results obtained from optimizing the application database. As data grows, there may be a drop in performance for access to the database shared by Biblio-MetReS and Homol-MetReS. That implies an increase in runtime and, therefore, a fall in QoS. In multiple situations, this entails noncompliance with the SLA agreement.

The current database server is located in a virtual machine, running on top of an HP Proliant DL165 G7 node with two Opteron 6,274 processors at $2.2 \mathrm{GHz}$ with 16 cores each $192 \mathrm{~GB}$ of DDR3 RAM and 4.5 TB. The resources assigned to the virtual machine are 4 cores, $32 \mathrm{~GB}$ of RAM, and $525 \mathrm{~GB}$ of disk.

6.1. Biblio-MetReS Optimization. To measure the effect of implementing a preprocessing strategy we carried out a set of experiments where the execution runtimes of Biblio-MetReS with and without the strategy were measured.

In the experiment, all the literature database sources were selected simultaneously for the search (see literature 


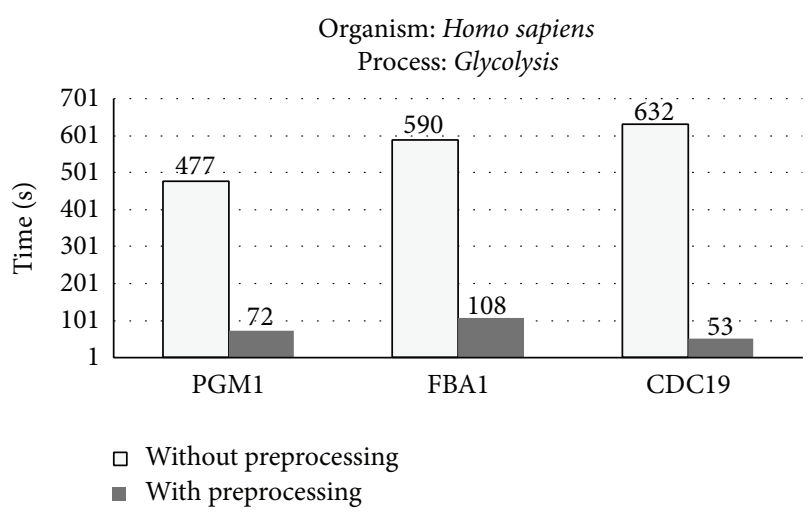

Figure 5: Runtimes with and without preprocessing for the PGM1, $F B A 1$, and CDC19 genes involved in the Glycolysis process of the Homo sapiens organism.

databases column in Figure 2). Figure 5 only shows the results obtained for the Homo sapiens organism. The PGM1, FBA1, and CDC19 genes involved in the Glycolysis process in Homo sapiens were used in the search. In the "without preprocessing" case, the runtime computed the search after the database had previously been emptied. During the procedure in doing the search, the database was updated with the statistical information of the documents found. Next, the same search was repeated (with the database containing almost all the documents). Thus, this operation shows the results "with preprocessing" activated. A large number of similar searches were done and the performance results were qualitatively similar (data not shown).

In general, performance gains, defined as the execution ratio between preprocessing and nonpreprocessing, ranged between 5 and 11, with an average improvement of 10. So, with preprocessing enabled, Biblio-MetReS was about 10 times faster than without preprocessing.

Figure 6 shows the response time evolution when retrieving different numbers of documents from the Doctable database table (from 1 to 700 documents). It can be seen how response time increased slightly from 0 to 0.06 seconds when accessing larger numbers of documents. However, these variations were not very significant as runtime was always below 0.1 seconds, and therefore it had no real impact on overall performance. So we can affirm that preprocessing is a consistent improvement in the performance of BiblioMetReS in the potential high-variability access to a datacenter like the one where this tool was hosted.

6.2. Optimal Database Parameters. Several performance tests based on the current database usage were carried out in order to determine the optimal values for the database. Table 6 shows a summary of the parameters thoroughly described in Section 5, containing their default values. Their optimal value (also in Table 6) was determined by using the MySQLTuner (https://github.com/major/MySQLTuner-perl) benchmark. Optimal values are referenced as "Optimized" and the default ones as "Default."

We were interested in how runtime was affected by optimizing the values of the parameter set shown in Table 6 in

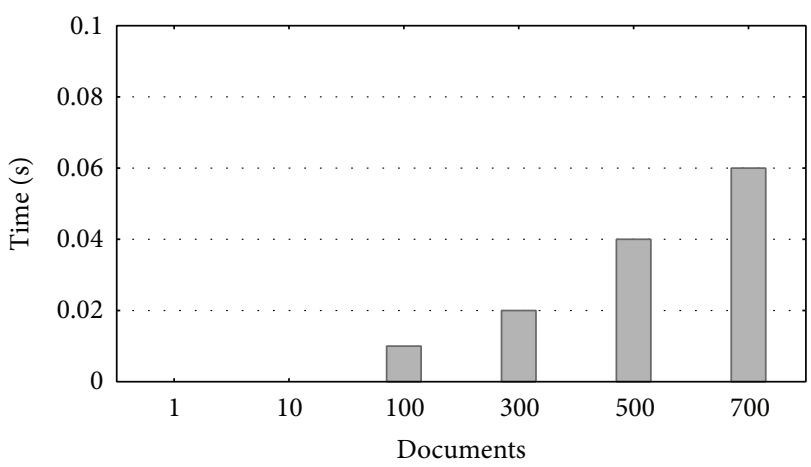

Figure 6: Runtime evolution when selecting from 1 to 700 simultaneous documents from the Doctable table.

the database server. In order to do the different optimization experiments, the current database server located in a virtual machine was replicated. Consequently, both database servers were located in their respective virtual machines, running on the HP Proliant DL165 G7 machine presented at the beginning of Results section.

Optimization of the parameters was done taking into account the database size, the maximum number of related tables in a search query in the database, and the length of tables and fields involved in it. These considerations led to the parameter limits shown in Table 6.

First, we determined the size of the database (6 GBytes), the main tables, and the fields to be used in the performance experiments. As a first optimization experiment we asked the biological question: "Is the distribution of protein sizes similar between organisms?" To answer this, we accessed each organism table and retrieved the size of the sequence of each annotated protein in its fully sequenced genome. The results are shown in Figure 7 . We can see that the statistical distribution of protein sizes between organisms is qualitatively similar and has a long tail. This operation created a dataset that allowed us to compare and build histograms for the sizes of all proteins between two organisms, YPN and SPM. YPN is the acronym for Yersinia pestis Nepal516 (biovar Antiqua) and SPM stands for Streptococcus pyogenes MGAS8232 (serotype M18). The $y p n \_I S O F O R M \_M A I N$ and spm_ISOFORM_MAIN tables had 4,094 and 1,839 entries, respectively. However, the average protein size is organism-specific and increases rapidly with the number of proteins contained in the genome.

The SQL query used in this occasion was the following:

SELECT OCTET_LENGTH (isoform_sequence) AS sequence,

COUNT(*) AS frequency

FROM hsa_ISOFORM_MAIN

GROUP BY OCTET_LENGTH

(isoform_sequence); 


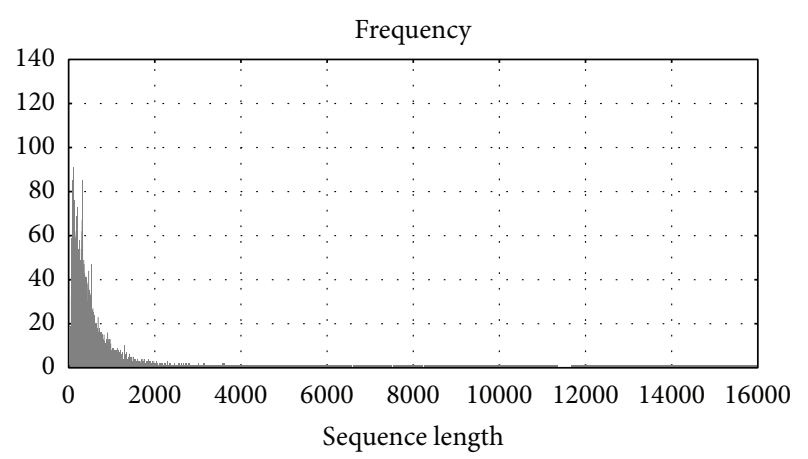

(a) Histogram HSA

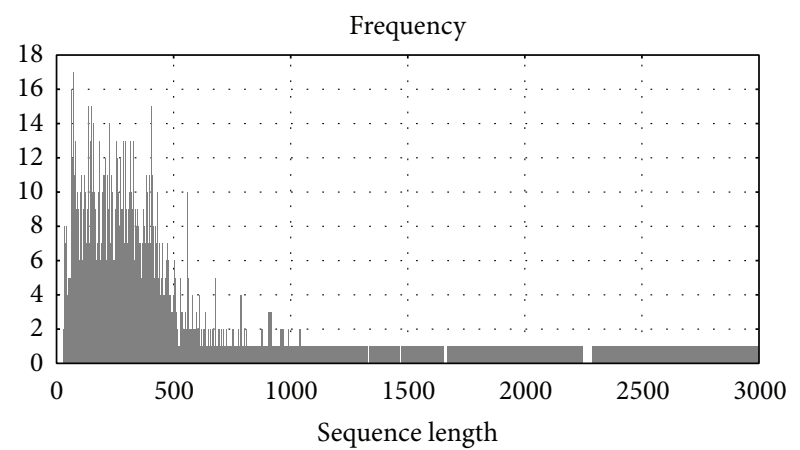

(b) Histogram AAC

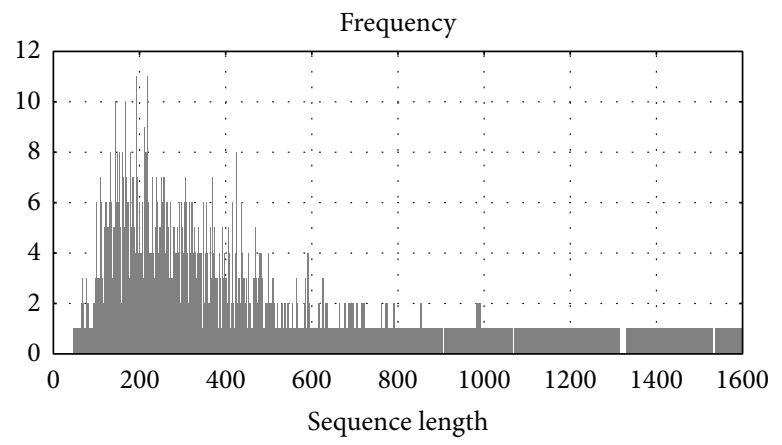

(c) Histogram AAE

FIGURE 7: Histograms.

TABLE 6: $M y S Q L$ optimization parameters.

\begin{tabular}{lcc}
\hline Variable & Default value & Optimized value \\
\hline query_cache_size & $0 \mathrm{MB}$ & $64 \mathrm{MB}$ \\
table_open_cache & 64 & 4096 \\
key_buffer_size & $7996 \mathrm{MB}$ & $100 \mathrm{MB}$ \\
join_buffer_size & $128 \mathrm{KiB}$ & $1 \mathrm{MB}$ \\
query_cache_limit & $1 \mathrm{MB}$ & $16 \mathrm{MB}$ \\
\hline
\end{tabular}

This kind of operation entails database searches of type (a) from Section 4 (retrieving information of the different organisms).

Experiments with the different parameter values of Table 6 were performed. Figure 8 summarizes the results of optimizing the values of some of the parameters from Table 6. The $x$-axis represents different combinations of the parameters and the $y$-axis measures the runtimes (in seconds). In the first column, all the parameters were set to their default values. In the other columns, the enumerated parameters were set to their optimal value, and the remaining parameters were unchanged from their default values. This way the impact of each parameter on the performance could be independently measured. The sensitivity of runtime to changes in each parameter was, in increasing order of importance, table_open_cache, join_buffer_size, query_cache_limit, and key_buffer_size. Generally speaking, it can be said that the use of the optimal parameter values had a great impact on the overall system performance. The query_cache_size parameter is discussed separately below.

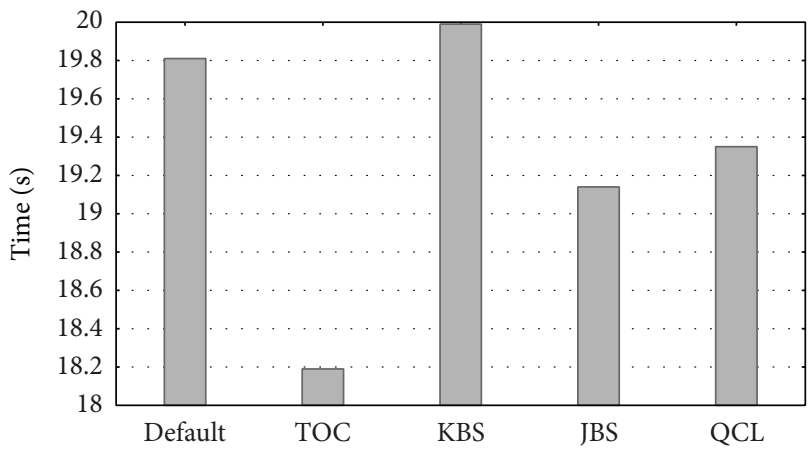

FIGURE 8: Runtimes for default and optimal values of the database server parameters. TOC: the table_open_cache, KBS: key_buffer_size, JBS: join_buffer_size, and QCL: query_cache_limit.

We have also manually analyzed the effect of changing parameter values on the performance of the system to confirm the good behavior of the automated optimization done using MySQLTuner. In general, we observed that further increases in the parameter values led to no improvement in the performance of the applications. As an example, the effect of query_cache_size on the performance is shown in Figure 9. In this case, the evolution of the runtime by ranging query_cache_size between the default and the optimal values is represented. We see that increasing the query cache size beyond $64 \mathrm{MB}$ had no effect on runtime, which justifies the choice of $64 \mathrm{MB}$ as the optimal value by the MySQLTuner benchmark. However, this optimal value should account for 


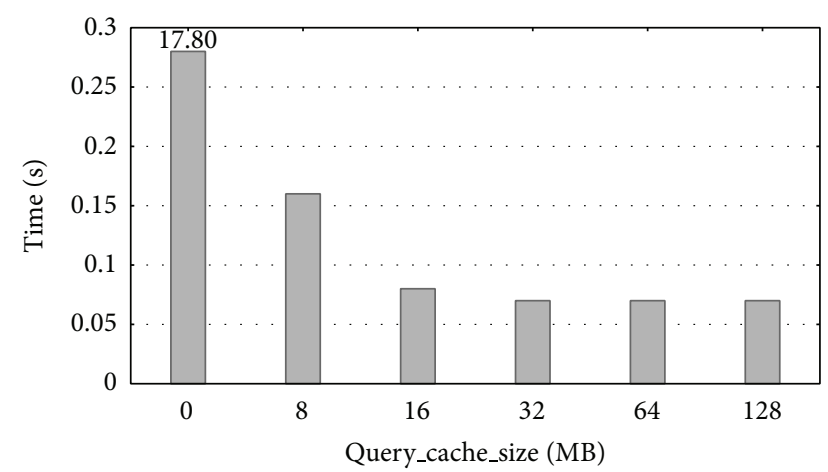

FIGURE 9: Runtime evolution when modifying the query_cache_size parameter.

database usage in order to avoid wasting resources. The $S Q L$ query used on this occasion was the following:

\section{SELECT COUNT $(*)$ FROM ypn_ISOFORM_MAIN LEFT JOIN (spm_ISOFORM_MAIN) ON (}

\author{
ypn_ISOFORM_MAIN. isoform_sequence = \\ spm_ISOFORM_MAIN. isoform_sequence);
}

The type of this database operation is (b) from Section 4 (comparing information data of multiple organisms).

We conclude this section by remarking that the SQL queries took on average 0.1441 seconds using the default parameter values and 0.002 seconds using the optimized parameter values. This shows that our proposed optimization can lead to improvements in runtime of approximately three orders of magnitude.

6.3. Stressing the Database. Next, the optimization effect was measured by running a representative set of queries in Bilio- and Homol-MetReS using the default parameter values. We then ran the same set of queries using the optimized parameter values. Figure 10 shows the runtime (in seconds) of the following $S Q L$ query used this time:

\section{SELECT * FROM Processestable;}

The type of this database operation is (a) from Section 4 (retrieving information about the different organisms). This operation determines the number of individual biological processes defined in the database. Table Processestable had 10,933 entries. It is a very common query when selecting the process in the Biblio-MetReS application in the query definition, done before choosing the genes/proteins (see Figure 2).

Figure 10 shows that runtimes using the optimized parameter values were slightly faster than when using the default parameter. However, the performance improvement was small, and this could hardly be appreciated by the final user when operating the applications.

Further experimentation was done with more complex queries to measure performance differences of both database's server configurations when more complex sets of

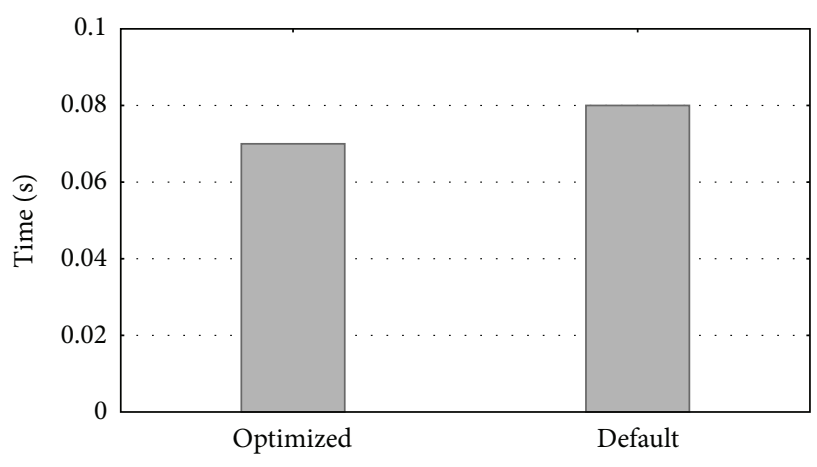

FIgURE 10: Runtime of a simple query.

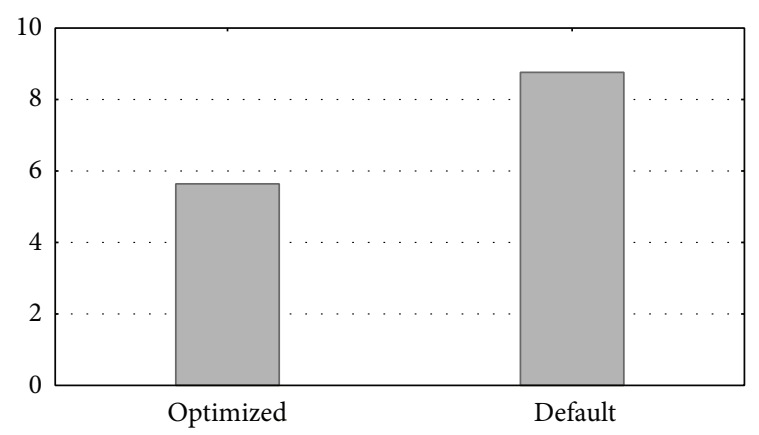

FIgURE 11: Runtime querying the Homo sapiens table.

operations are performed. Figure 11 shows an example of such a search. Here, we can see a higher performance difference between the runtime of a query accessing a single table when using the optimized and the default parameter values.

In this case, the $S Q L$ query was the following:

\section{SELECT isoform_sequence FROM hsa_ISOFORM_MAIN;}

The type of this database operation is (a) from Section 4 (retrieving information about the different organisms).

This experiment selected the sequences of all the proteins in the proteome of the Homo sapiens organism. The hsa_ISOFORM_MAIN table used had a total length of 25,796 entries. This is a common type of search in the database performed by users in both the Biblio- and Homol-MetReS applications. This operation is especially critical in the HomolMetReS application, because similarities between genes are found by comparing the sequences retrieved from such a table with all the organisms. These comparisons give additional information or suggestions about organisms that lack this gene data. On this occasion, the gains of the optimized database compared to the nonoptimized one were quite significant (see Figure 11). We performed similar systematic searches in accessing other organism tables and the results were qualitatively similar to those shown in Figure 11 (data not shown).

As stated above, Homol-MetReS compares sequences of protein between two organisms. To do so, the application needs to simultaneously access two tables. An example of the effect of parameter optimization on such operations is shown 


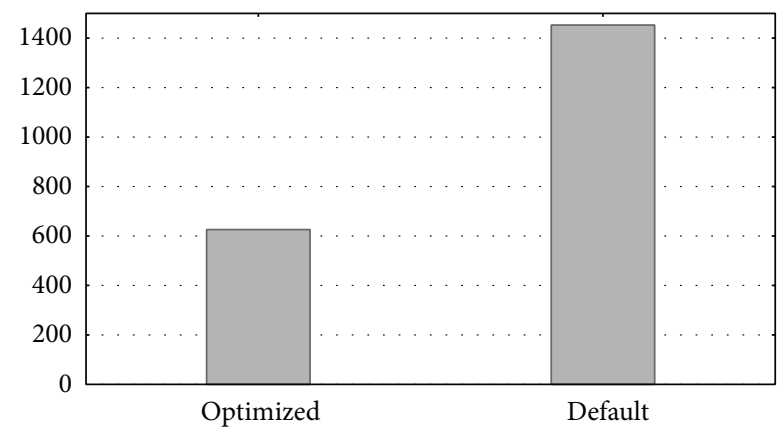

Figure 12: Runtime of a left join using 2 tables.

in Figure 12. This figure shows the runtime of a query that accesses two tables simultaneously. This operation creates a dataset that allows us to compare and build histograms for the sizes of all proteins between two organisms, ZMA and XLA. ZMA is the acronym for Zea mays (maize) and XLA stands for Xenopus laevis (African clawed frog).

The SQL query from Figure 12 is the following:

SELECT zma_ISOFORM_MAIN.
isoform_sequence FROM zma_ISOFORM_MAIN
LEFT JOIN (xla_ISOFORM_MAIN) ON (
zma_ISOFORM_MAIN . isoform_sequence =
xla_ISOFORM_MAIN .isoform_sequence);

The type of this database operation is (b) from Section 4 (comparing information data between multiple organisms).

The tables used in this experiment, zma_ISOFORM MAIN and xla_ISOFORM,_MAIN, contain 17,821 and 10,681 entries, respectively. The $S Q L$ operation is a LEFTJOIN, which is very expensive computationally speaking. This experiment shows how, even in extreme stressed scenarios, the optimization of parameters leads to a strong performance improvement that decreases runtime by more than $50 \%$. These results illustrate that our approach to optimizing database access parameters can lead to significant improvements in performance when running operations in HomolMetReS and, to a lesser degree, in Biblio-MetReS.

6.4. Searching with Preprocessing. Typically, the most timeconsuming operations in Biblio-MetReS do not involve database access. Rather, they have to do with on-the-fly analysis of text in scientific documents. As stated above, improving the performance of the application for these operations was done by implementing a preprocessing strategy, as seen in Section 3. Nevertheless, as the number of preprocessed documents contained in the application's database increases, the effect of optimizing database access on the performance of the application will be increasingly significant. Therefore, it is important to estimate what the effect of optimizing database access might be in the future. To do so, we perform Biblio-MetReS-related searches using default versus optimized parameter values. The searches are done by selecting all defined biological processes from the table Processpairstable, which contains 33,731 entries.

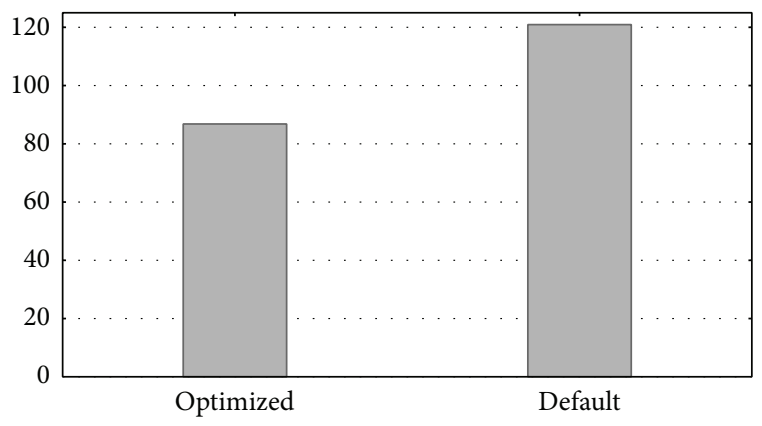

FIGURE 13: Runtime of a search query to the Processpairstable table.

Figure 13 shows that optimization of parameter values leads to significant improvements of about $30 \%$ in the runtime of this type of search.

The SQL query from Figure 13 is the following:

\section{SELECT * FROM Processpairstable;}

The type of this database operation is (a) from Section 4 (retrieving information about the different organisms).

6.5. MySQL versus MapReduce. We were interested in quantifying the effect of using an optimized regular relational database implemented in $M y S Q L$ versus the utilization of a NoSQL database, when the amount of available data is below the terabyte limit. To do so, we compared the performance of a set of searches run against our optimized database versus the same set of searches run against equivalent NoSQL implementation of our database. This implementation was done with MapReduce and HBase. MapReduce is a programming model for processing large datasets with a parallel, distributed algorithm on a supercomputing system (i.e., large cluster system). HBase is thought for random and realtime read/write access to large amount of data. The new database was mapped in another virtual machine with the same computational resources as the original database. Consequently, the two database servers were located in their respective virtual machines, running on the HP Proliant DL165 G7 machine presented at the beginning of Results section.

In the experiment, the Processestable table was accessed to compare the response times of a commonly used query on both systems. Figure 14 shows the runtime (in seconds) of the following $S Q L$ query used this time:

\section{SELECT $*$ FROM Processestable;}

In summary, $M y S Q L$ obtained the best performance. $M y S Q L$ was one order of magnitude faster than MapReduce/HBase (averaged 60\% faster). This justifies the selection of a typical database like $M y S Q L$ as the technology for implementing the database server.

\section{Discussion}

The optimization experiments presented have been widely tested in order to determine the parameter values that 


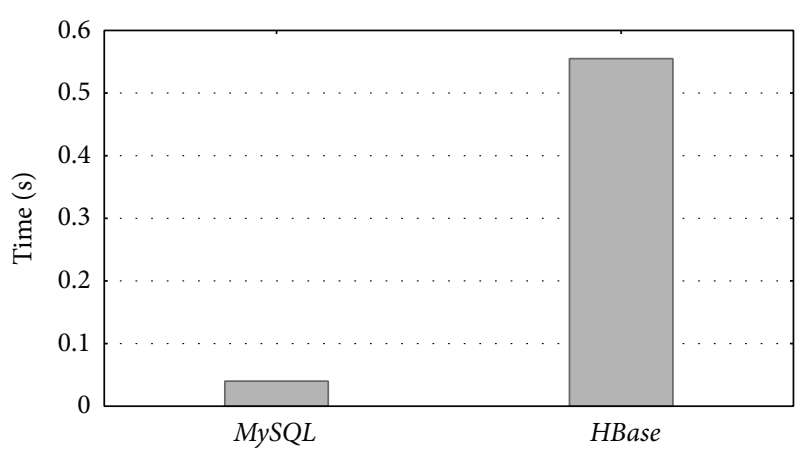

FIgURE 14: MySQL and MapReduce runtimes when accessing the Processestable table.

maximize application performance. We present the main results and relate gains obtained with the kind of operations performed by Biblio-MetReS and Homol-MetReS. The optimal tunable values for the search in the database server were found empirically. The application whose performance was more extensively improved was Homol-MetReS.

A special search affecting the preprocessing was analyzed. Preprocessing reduced 10 times the Biblio-MetReS runtime. In addition, our proposed optimization can lead to improvements of three orders of magnitude. Even in extreme stressed scenarios, the optimization decreased runtime by more than $50 \%$.

We also compared the performance of the database server (implemented as a relational MySQL database) against the same database implemented with the MapReduce in order to verify that the database was not large enough to apply NoSQL technologies. MySQL averaged $60 \%$ faster than MapReduce/HBase.

\section{Conclusions}

In this paper, we analyzed and optimized the performance of two biological cloud applications. We presented one main solution to achieve this improvement that consisted of analyzing and optimizing the database access parameters used by both applications in order to obtain a better level of performance. We successfully analyzed the current database demands and determined the parameters that could provide a greater positive impact on the system performance. The results show a significant improvement in the runtime of some of the most common and significant queries in a MySQL database, done by the biological applications presented, Biblio- and Homol-MetReS. The fully sequenced database used needs sizes of one or more orders of magnitude bigger than the one used by the biological applications used in this work to justify its use in NoSQL technologies. Preprocessing can further extend the use of $S Q L$ databases. Although this process was effective enough to obtain an acceptable performance, a NoSQL approach may be needed in the future as the database grows both in concurrent queries and size as more fully sequenced organism genomes are added.

\section{Conflict of Interests}

The authors declare that there is no conflict of interests regarding the publication of this paper.

\section{Acknowledgments}

This work was supported by the MEyC under Contracts TIN2011-28689-C02-02 and BFU20 10-17704. The authors are members of the research groups 2009-SGR145 and 2009SGR809, funded by the Generalitat de Catalunya.

\section{References}

[1] A. Usié, H. Karathia, I. Teixidó et al., "Biblio-MetReS: a bibliometric network reconstruction application and server," $B M C$ Bioinformatics, vol. 12, article 387, 2011.

[2] H. Karathia, E. Vilaprinyo, A. Sorribas, and R. Alves, "Saccharomyces cerevisiae as a model organism: a comparative study," PLoS ONE, vol. 6, no. 2, Article ID e16015, 2011.

[3] A. Barbosa-Silva, T. G. Soldatos, I. L. F. Magalhães et al., "LAITOR-literature assistant for identification of terms co-occurrences and relationships," BMC Bioinformatics, vol. 11, article 70, 2010.

[4] R. Hoffmann and A. Valencia, "Implementing the iHOP concept for navigation of biomedical literature," Bioinformatics, vol. 21, no. 2, pp. 252-258, 2005.

[5] D. Szklarczyk, A. Franceschini, M. Kuhn et al., "The STRING database in 2011: functional interaction networks of proteins, globally integrated and scored," Nucleic Acids Research, vol. 39, no. 1, pp. D561-D568, 2011.

[6] B. P. Kelley, B. Yuan, F. Lewitter, R. Sharan, B. R. Stockwell, and T. Ideker, "PathBLAST: a tool for alignment of protein interaction networks," Nucleic Acids Research, vol. 32, pp. W83W88, 2004.

[7] S. Chaudhury and O. Igoshin, "Dynamic disorder-driven substrate inhibition and bistability in a simple enzymatic reaction," The Journal of Physical Chemistry B, vol. 113, no. 40, pp. 1342113428, 2009.

[8] J. W. Veening, O. A. Igoshin, and O. P. Kuipers, “Transient heterogeneity in extracellular protease production by Bacillus subtilis," Molecular Systems Biology, vol. 4, article 184, 2008.

[9] K. Xiong and H. Perros, "Service performance and analysis in cloud computing," in Proceedings of the 5th World Conference on Services, pp. 693-700, Los Angeles, Calif, USA, September 2009.

[10] J. Martin and A. Nilsson, "On service level agreements for IP networks," in Proceedings of the IEEE INFOCOM, pp. 855-863, June 2002.

[11] K. Xiong and H. Perros, "Service performance and analysis in cloud computing," in Proceedings of the World Conference on Services-I, pp. 693-700, Los Angeles, Calif, USA, July 2009.

[12] R. Aversa, B. Di Martino, M. Rak, S. Venticinque, and U. Villano, "Performance prediction for HPC on clouds," in Cloud Computing: Principles and Paradigms, John Wiley \& Sons, 2011.

[13] D. Abadi, "Data management in the cloud: limitations and opportunities," IEEE Data Engineering Bulletin, vol. 32, no. 1, article 312, 2009.

[14] F. Chang, J. Dean, S. Ghemawat et al., "Bigtable: a distributed storage system for structured data," in Proceedings of the 7th 
USENIX Symposium on Operating Systems Design and Implementation (OSDI '06), Amazon's Dynamo, Seattle, Wash, USA, November 2006.

[15] G. DeCandia, D. Hastorun, M. Jampani et al., "Dynamo: amazon's highly available key-value store," in Proceedings of the 21st ACM SIGOPS Symposium on Operating Systems Principles (SOSP '07), pp. 205-220, Stevenson, Wash, USA, 2007.

[16] A. Lakshman and P. Malik, "Cassandra-a Decentralized Structured Storage system," in Proceedings of the Large Scale Distributed Systems and Middleware Workshop (LADIS '09), 2009.

[17] Project Voldemort, http://project-voldemort.com/.

[18] O. C. Lin, A. T. Kwee, and F. S. Tsai, "Database optimization for novelty detection," in Proccedings of the 7th International Conference on Information, Communications and Signal Processing (ICICS '09), pp. 1-5, December 2009.

[19] C. Bujdei, S.-A. Moraru, and S. Dan, "Optimize databases for health monitoring systems," in Proceedings of the 1st International Conference on Pervasive Technologies Related to Assistive Environments (PETRA '08), ACM, July 2008.

[20] T. Jin, X. Xu, J. Fang et al., "How human leukocytes track down and destroy pathogens: lessons learned from the model organism Dictyostelium discoideum," Immunologic Research, vol. 43, no. 1-3, pp. 118-127, 2009.

[21] N. Siva, "1000 Genomes project," Nature Biotechnology, vol. 26, no. 3, p. 256, 2008.

[22] H. Ellegren, "Comparative genomics and the study of evolution by natural selection," Molecular Ecology, vol. 17, no. 21, pp. 45864596, 2008.

[23] H. Tettelin, D. Riley, C. Cattuto, and D. Medini, "Comparative genomics: the bacterial pan-genome," Current Opinion in Microbiology, vol. 11, no. 5, pp. 472-477, 2008.

[24] R. Alves, E. Vilaprinyo, and A. Sorribas, "Integrating bioinformatics and computational biology: perspectives and possibilities for in silico network reconstruction in molecular systems biology," Current Bioinformatics, vol. 3, no. 2, pp. 98-129, 2008. 

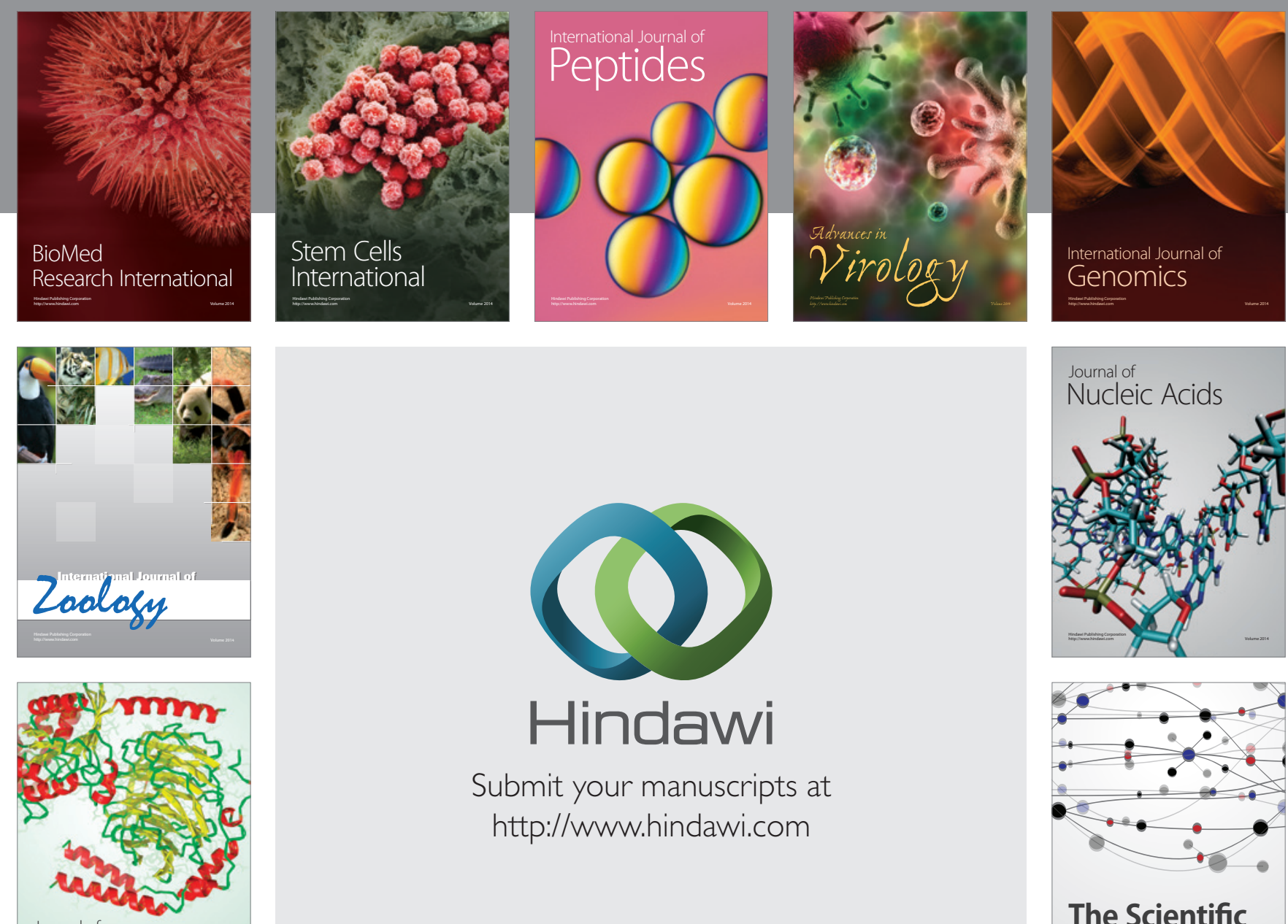

Submit your manuscripts at

http://www.hindawi.com

Journal of
Signal Transduction
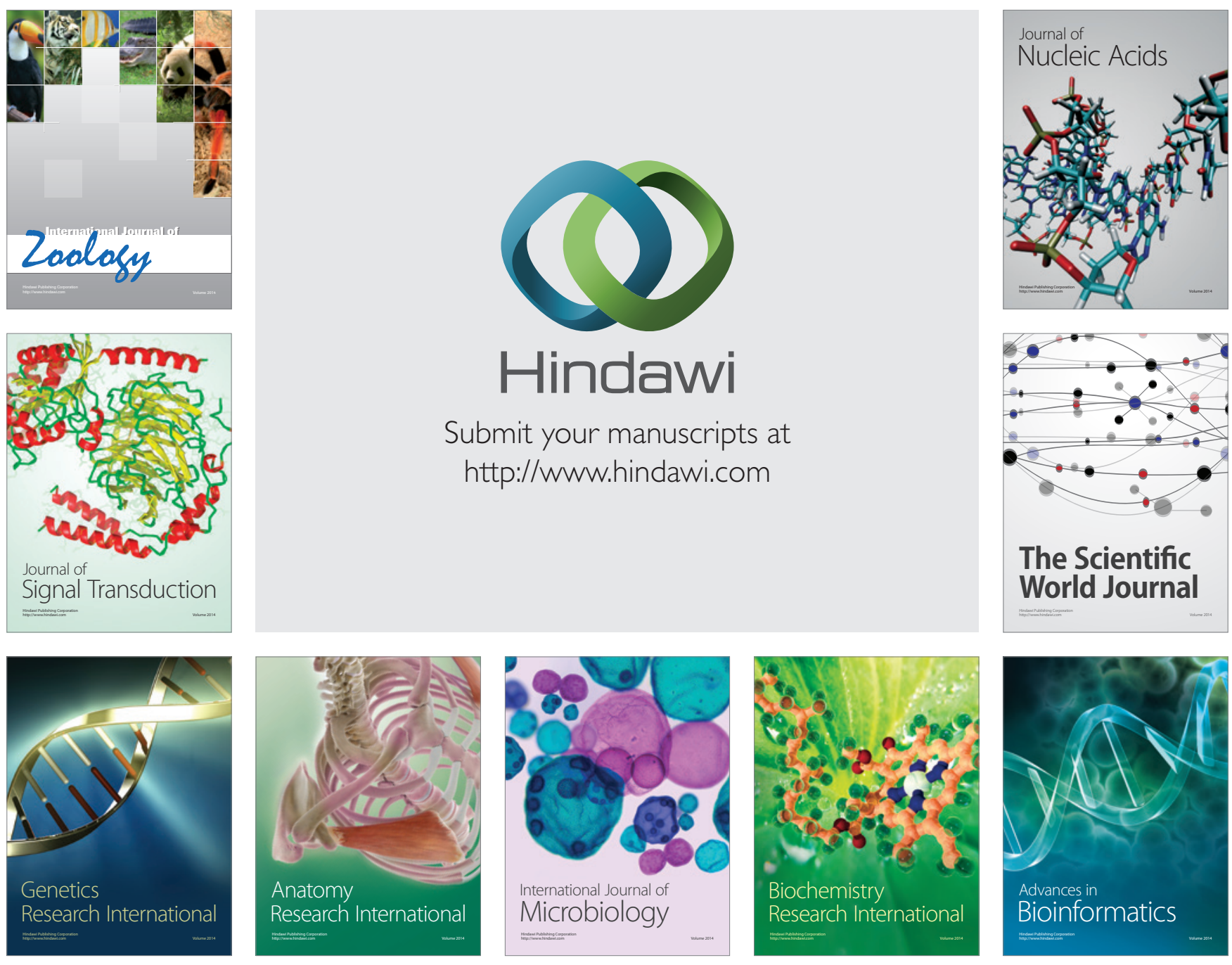

The Scientific World Journal
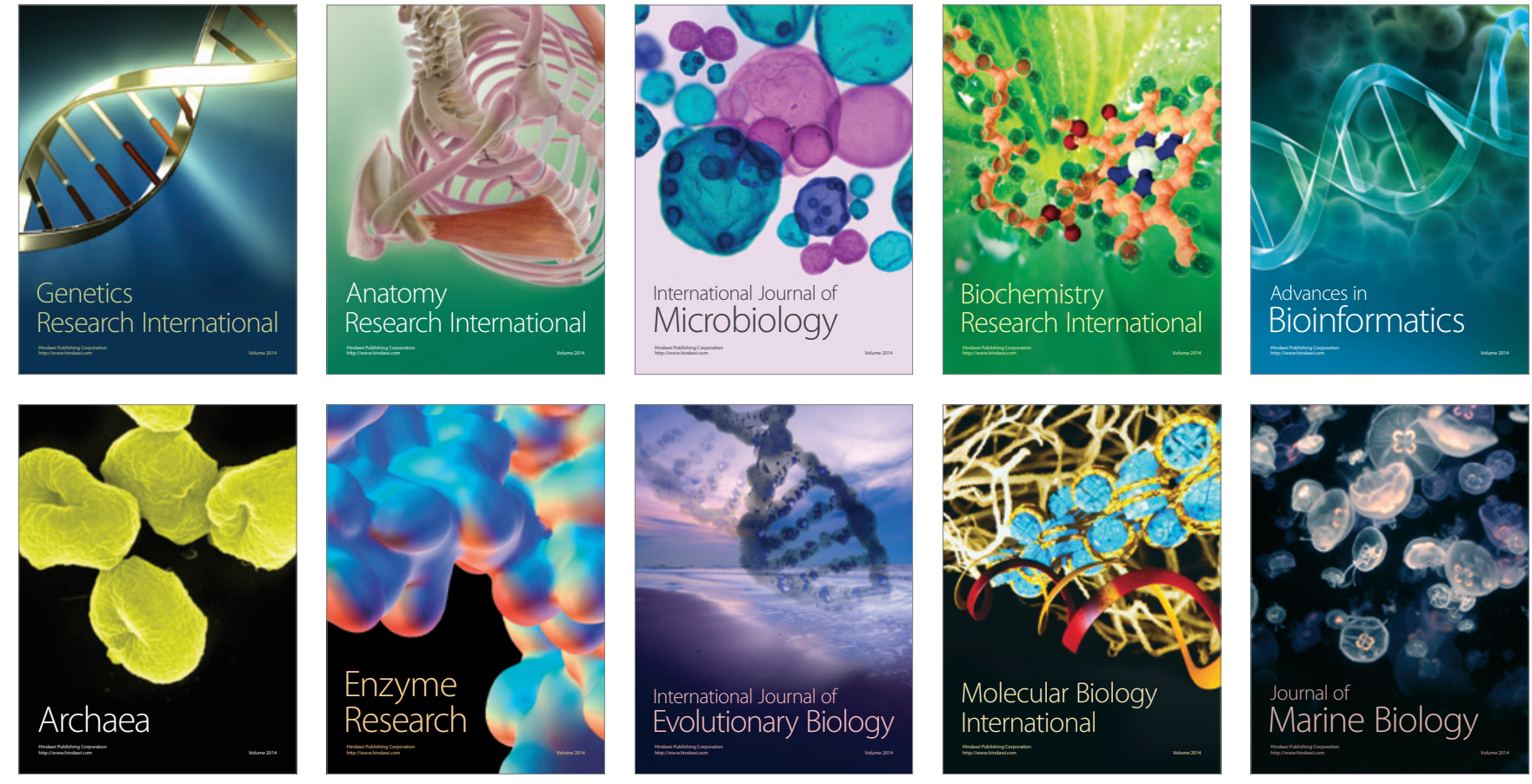